\title{
Higher-order mode rf guns
}

\author{
John W. Lewellen \\ Advanced Photon Source, 401/B2207, Argonne National Laboratory, Argonne, Illinois 60439
}

(Received 27 February 2001; published 30 April 2001)

\begin{abstract}
Traditional photocathode rf gun design is based around the use of $\mathrm{TM}_{0,1,0}$-mode cavities. This is typically done in the interest of obtaining the highest possible gradient per unit supplied rf power and for historical reasons. In a multicell, aperture-coupled photoinjector, however, the gun as a whole is produced from strongly coupled cavities oscillating in a $\pi$ mode. This design requires very careful preparation and tuning, as the field balance and resonant frequencies are easily disturbed. Side-coupled designs are often avoided because of the dipole modes introduced into the cavity fields. This paper proposes the use of a single higher-order mode rf cavity in order to generate the desired on-axis fields. It is shown that the field experienced by a beam in a higher-order mode rf gun is initially very similar to traditional 1.5- or 2.5-cell $\pi$-mode gun fields, and projected performance in terms of beam quality is also comparable. The new design has the advantages of much greater ease of fabrication, immunity from coupledcell effects, and simpler tuning procedures. Because of the gun geometry, the possibility also exists for improved temperature stabilization and cooling for high duty-cycle applications.
\end{abstract}

DOI: $10.1103 /$ PhysRevSTAB.4.040101

PACS numbers: 41.75.Ht, 41.75.Fr, 29.25.Bx

\section{INTRODUCTION}

A split-type photoinjector consists of a photocathode rf gun, a focusing and emittance compensation solenoid, a drift distance, and at least one capture linac section. The gun itself is typically a standing-wave, coupled-cavity resonator made from two or more cylindrical cells. If the gun has a design resonant frequency of $f_{\text {rf }}$, corresponding to a free-space wavelength of $\lambda_{\text {rf }}$, a "full" cell typically has a length of $\lambda_{\text {rf }} / 2$. The cathode is located in a "fractional" cell, with length usually on the order of $0.5-0.6$ times the length of a full cell. Typically, power is fed in through the wall of a full cell via waveguide and flows to the other cell(s) via on-axis apertures, although alternate schemes have been proposed and demonstrated [1]. Each cell is nominally a $\mathrm{TM}_{0,1,0}$-mode cavity, and the coupling between the cells is designed to have the peak on-axis electric fields approximately equal in all cells. An example of this type of gun design is shown in Fig. 1. This particular gun is an $S$-band gun generated by a BNL/SLAC/UCLA collaboration, and will be referred to hereafter as a BNL-type gun. Figure 1 also shows an overlay of a $\mathrm{TM}_{0,1,2}$-mode cavity.

The BNL-type gun design has proven to be reasonably effective in the generation of high-brightness electron beams [2,3]. However, there are two main disadvantages. First, because the design consists of a series of strongly coupled resonators, extreme care must be taken in the fabrication and tuning of the gun. Each cell must be tuned independently and then adjusted for the desired field balance. Because the cavities are not true $\mathrm{TM}_{0,1,0}$-mode cavities (due to the presence of the coupling and output beam apertures), even small variations in cavity geometries can have a strong affect on the actual field produced. Second, the aperture region between the cells can experience very strong fields when the guns are operated at high on-axis gradients. This can result in arcing and local heating problems at the aperture. In turn, this can lead to operational difficulties such as cavity detuning.

It is possible, however, to design a single-cell resonator having an on-axis field profile that closely approximates a standard multicell photoinjector. This has several advantages. First, fabrication would be greatly eased, as the basic cavity geometry is extremely simple. Second, as the entire gun consists of a single cell, all concerns arising out of the use of multiple cells (intercell coupling, frequency pulling, etc.) are eliminated. Finally, the design eliminates all nose cones internal to the gun. In addition to the mentioned increase in fabrication ease, this should help provide for improved cooling for the gun.

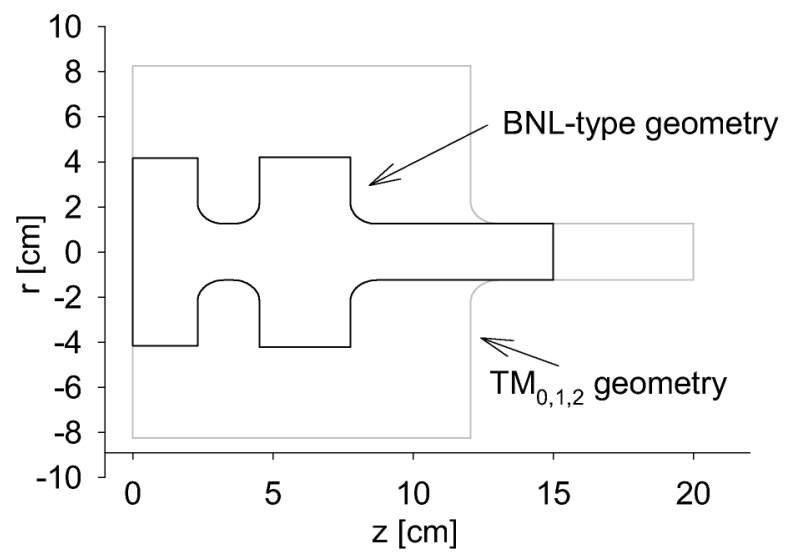

FIG. 1. Geometry for the cylindrically symmetric BNL-type, 1.6-cell rf gun. The overlay shows the geometry of a $\mathrm{TM}_{0,1,2^{-}}$ mode cavity at the same resonant frequency. 


\section{CAVITY DESIGN}

The resonant frequencies of a true $\mathrm{TM}_{0, m, n}$ cavity are given by the formula [4]

$$
\omega_{0, m, n}=\frac{1}{\sqrt{\mu_{0} \varepsilon_{0}}} \sqrt{\left(\frac{r_{m}}{R}\right)^{2}+\left(\frac{n \pi}{d}\right)^{2}},
$$

where $\omega$ is the resonant frequency of the cavity in $\mathrm{rad} / \mathrm{sec}$, $r_{m}$ is the $m$ th root of the $J_{0}$ Bessel function, $R$ is the cavity radius, $d$ is the cavity length, $m$ is the number of radial variations in the sign of the field, and $n$ is the number of longitudinal variations in the sign of the field. (We consider here only fields with no azimuthal dependence.) In a pure $\mathrm{TM}_{0, m, n}$ cavity, the on-axis electric field is always at a maximum at $z=0$ and $z=d$. If a beam exit aperture is placed at $z=d$, however, the on-axis field will gradually taper off to zero. A $\mathrm{TM}_{0,1,2}$-mode cavity, thus modified with a beam exit aperture, should have an on-axis field profile not dissimilar to a standard 1.5-cell gun of the same resonant frequency, for appropriately chosen values of $R$ and $d$. Consider Fig. 2, which compares the on-axis field profile of a standard $S$-band BNL-type gun with a $\mathrm{TM}_{0,1,2}$-mode cavity including a beam exit port. The field of the $\mathrm{TM}_{0,1,2}$ cavity has two longitudinal variations in sign; if one were to attempt to construct this field profile using a coupled-cavity design, the cell order would be $0.5-1-0.5$ cells.

The major difference in terms of the field seen by a beam is the presence, in the field of the higher-order mode (HOM) cavity, of what is effectively an additional 0.5-cell cavity at the end of the gun. Also, the longitudinal field of the HOM cavity decreases slightly faster off the cathode than that of the BNL-type gun, which leads to slightly less rf focusing off the cathode; this can be compensated by small changes in the cathode wall. In addition, the equivalent of the field balance between the cells can be altered significantly by changing the slope of the outer wall, e.g., making the cavity a truncated cone rather than a cylinder.

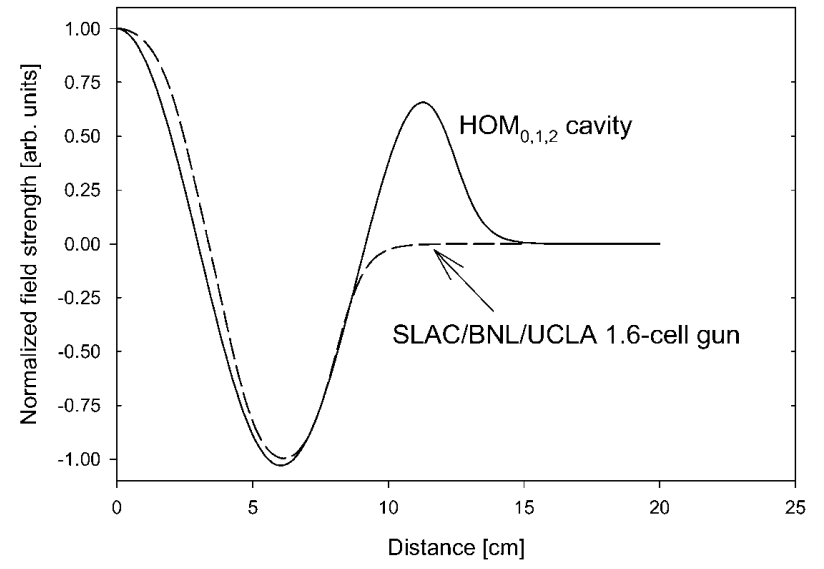

FIG. 2. On-axis fields for the BNL-type gun and the $\mathrm{TM}_{0,1,2}$ cavity, generated using the geometry shown in Fig. 1.

\section{CAVITY AND RF PROPERTIES}

Overall, the HOM-type cavity rf properties are quite reasonable and in many ways comparable to the BNLtype gun design. All of the parameters presented were calculated using SUPERFISH and use the cavity geometry shown in Fig. 1.

Mode separation is excellent. For cavity dimensions of $8.25 \mathrm{~cm}$ radius, $12.05 \mathrm{~cm}$ length, and exit beam pipe radius of $1.25 \mathrm{~cm}$, the next nearest supported modes are more than $100 \mathrm{MHz}$ away. [This can be seen from Eq. (1); note, however, that the presence of the exit beam aperture causes significant frequency pulling. SUPERFISH frequency sweeps show fewer supported modes than indicated by Eq. (1).] The radius of the cavity is about twice that of a typical $S$-band gun, but it is still within reasonable limits as far as practical construction is concerned. The increased radius should also provide for more stored rf energy if pulse-train operation is desired.

The cavity $Q$, at 39500 , is more than twice that of the BNL-type gun at 15900 . The ratio of peak on-axis field to peak surface field is roughly the same for both guns. The ratio of peak on-surface magnetic field to peak on-axis electric field is about $3160 \mathrm{~A} / \mathrm{MV}$ for the HOM gun cavity, while for the BNL-type cavity the ratio is about $1830 \mathrm{~A} / \mathrm{MV}$. So, for the same on-axis field, the HOM-type cavity will see a higher peak wall current.

A decided disadvantage of the HOM cavity is the reduced shunt impedance. For the cavity geometry shown in Fig. 1, for instance, the shunt impedance is $13.6 \mathrm{M} \Omega / \mathrm{m}$, compared to a shunt impedance of approximately $68 \mathrm{M} \Omega / \mathrm{m}$ for the BNL-type gun design. As a result, the HOM-type cavity also has a higher total power requirement, by about a factor of 7 , for similar on-axis peak gradients. The HOM-type cavity radius is approximately twice that of the BNL-type, so the average power density dissipated on the surface of the gun is higher only by a factor of about 4 . Since the HOM cavity has no internal protrusions such as nose cones, however, cavity cooling for high-duty-factor operation should be easier than for a more traditional design. Also, it is relatively easy to improve the shunt impedance by, for instance, changing the outer wall shape from a cylinder to a truncated cone. This technique can also be used to alter the effective "cell length" and field strength ratios in the gun.

TABLE I. Parameter comparison between the BNL/SLAC/ UCLA and HOM gun designs; some parameters are approximate or average.

\begin{tabular}{lcc}
\hline \hline \multicolumn{1}{c}{ Parameter } & BNL-type & HOM-type \\
\hline Cell wall radius $(\mathrm{cm})$ & 4.2 & 8.2 \\
Interior length $(\mathrm{cm})$ & 7.7 & 12 \\
$Q$ & 15900 & 39500 \\
$B_{\text {peak }} / E_{\text {peak }}(\mathrm{A} / \mathrm{MV})$ & 1830 & 3160 \\
Shunt impedance $(\mathrm{M} \Omega) / \mathrm{m}$ & 68 & 13.6 \\
\hline \hline
\end{tabular}


A comparison of $\mathrm{rf}$ and basic cavity parameters is presented in Table I. The table lists the parameters for the most basic HOM-type design, e.g. without any outer wall taper, cathode plane geometry changes, etc.

\section{SIMULATED PERFORMANCE}

Initial simulation results of testing HOM cavities as photoinjectors are very encouraging. PARMELA version 2 was used to perform all of the simulations with beam. Figure 3 shows the simulated performance of an $S$-band $\mathrm{HOM}_{0,1,2}$ cavity rf gun with slight variations in back wall geometry, as a function of solenoid strength. The average on-axis gradient is chosen to be $47 \mathrm{MV} / \mathrm{m}$. This yields a peak on-axis field of about $120 \mathrm{MV} / \mathrm{m}$. The exit beam energy of 7.0-7.4 MeV (depending on backplane geometry) is higher than an equivalent 1.5-cell BNL-type gun, however, due to the presence of the effective additional half-cell at the gun exit. The bunch charge was taken to be $1 \mathrm{nC}$, with a flattop transverse emission radius of $1 \mathrm{~mm}$, uniform emission over $10 \mathrm{ps}$, and centroid launch phase of $35^{\circ}$ after the electric field zero crossing. The solenoid is simulated via a series of coils and is roughly based on the BNL-type solenoid. It has a FWHM of approximately $19 \mathrm{~cm}$, a coil pack starting at the exit of the gun, and zero on-axis field at the cathode plane via a bucking solenoid. The total distance between the cathode and the entrance to the capture linac section was $105 \mathrm{~cm}$, and the linac gradient was $7 \mathrm{MV} / \mathrm{m}$.

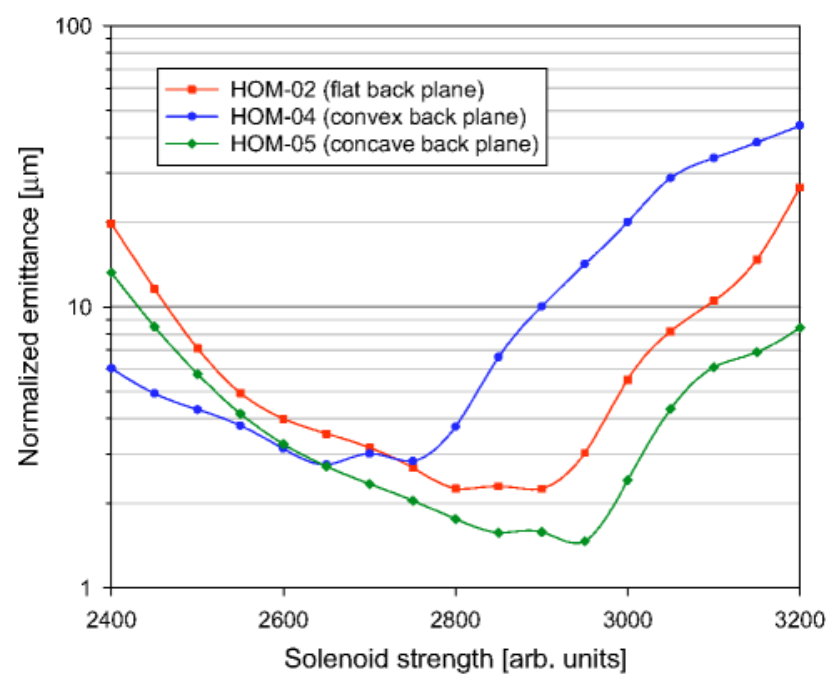

FIG. 3. (Color) Simulated performance of the $\mathrm{TM}_{0,1,2}$-mode cavity as a photoinjector, for three small variations in the cathode plane geometry.
The whole-beam emittances shown are measured immediately following the capture linac section.

The simple changes to the backplane geometry of the gun demonstrate the easy tunability presented by this design, and with only this modest amount of tuning the whole-beam emittance numbers are already very good. These emittance values can probably be further improved.

\section{CONCLUSIONS}

Higher-order mode cavities appear to offer a potentially viable and considerably simpler path toward photocathode gun production. The field profiles in the cavity, which are critical to proper operation of the gun, may be easily altered via simple changes in the cavity outer wall and backplane. Initial simulations have demonstrated that a gun made from a single HOM cavity should be capable of producing very high quality electron beams. The design offers immunity from coupled-cell effects, good mode separation, and the possibility of lower production cost and enhanced high-duty-factor operation because of the greatly simplified internal geometry. The primary disadvantage at this time would appear to be the higher rf power requirements for a HOM cavity gun. As the total energy gain per unit average on-axis gradient is higher with the HOM-type gun than the BNL-type gun, this may not prove to be an insurmountable difficulty, and the shunt impedance and longitudinal field profile have been found to be very amenable to improvement by small cavity profile changes.

\section{ACKNOWLEDGMENTS}

The author thanks Stephen Milton, John Noonan, John Schmerge, and Gil Travish for their thoughts and comments. This work is supported by the U.S. Department of Energy, Office of Basic Energy Sciences, under Contract No. W-31-109-ENG-38.

[1] L. C.-L. Lin, S. C. Chen, and J. S. Wurtele, Nucl. Instrum. Methods. Phys. Res., Sect. A 384, 274-284 (1997).

[2] D. T. Palmer et al., in Proceedings of the 1997 Particle Accelerator Conference, Vancouver, Canada, edited by M. Comyn (IEEE, Piscataway, NJ, 1998), pp. 2687-2689.

[3] D. T. Palmer et al., in Towards X-Ray Free Electron Lasers, edited by R. Bonifacio and W. A. Barletta, AIP Conf. Proc. No. 413 (AIP, New York, 1997), pp. 155-168.

[4] J.D. Jackson, Classical Electrodynamics (Wiley, New York, 1975), 2nd ed., p. 355. 